\title{
The APOA5 Trp 19 allele is associated with metabolic syndrome via its association with plasma triglycerides
} Jean Dallongeville*1, Dominique Cottel ${ }^{1}$, Aline Wagner ${ }^{2}$, Pierre Ducimetière ${ }^{3}$, Jean-Bernard Ruidavets ${ }^{4}$, Dominique Arveiler ${ }^{2}$, Annie Bingham ${ }^{3}$, Jean Ferrières ${ }^{4}$, Philippe Amouyel ${ }^{1}$ and Aline Meirhaeghe ${ }^{1}$

Address: ${ }^{1}$ Institut Pasteur de Lille, INSERM, U744, Université de Lille 2, Lille, France, ${ }^{2}$ EA 1801, Laboratoire d'Epidémiologie et Santé Publique, Université Louis Pasteur, Faculté de Médecine, Strasbourg, France, ${ }^{3}$ INSERM, U780, Villejuif, Université Paris-Sud XI, France and ${ }^{4}$ INSERM, U558, Toulouse, Faculté de Médecine, Université Paul Sabatier, Toulouse, France

Email: Jean Dallongeville* - jean.dallongeville@pasteur-lille.fr; Dominique Cottel - dominique.cottel@pasteur-lille.fr; Aline Wagner - Aline.Wagner@medecine.u-strasbg.fr; Pierre Ducimetière - ducimeti@vjf.inserm.fr; Jean-Bernard Ruidavets - ruidavet@cict.fr; Dominique Arveiler - monica@medecine.u-strasbg.fr; Annie Bingham - bingham@vjf.inserm.fr; Jean Ferrières - ferriere@mail.cict.fr; Philippe Amouyel - philippe.amouyel@pasteur-lille.fr; Aline Meirhaeghe - aline.meirhaeghe-hurez@pasteur-lille.fr

* Corresponding author

Published: 12 September 2008

BMC Medical Genetics 2008, 9:84 doi:10.1 186/147|-2350-9-84
Received: 21 January 2008

Accepted: 12 September 2008

This article is available from: http://www.biomedcentral.com/I47I-2350/9/84

(C) 2008 Dallongeville et al; licensee BioMed Central Ltd.

This is an Open Access article distributed under the terms of the Creative Commons Attribution License (http://creativecommons.org/licenses/by/2.0), which permits unrestricted use, distribution, and reproduction in any medium, provided the original work is properly cited.

\begin{abstract}
Background: The goal of the present study was to assess the effect of genetic variability at the APOA5/A4/C3/AI cluster locus on the risk of metabolic syndrome.

Methods: The APOA5 Ser19Trp, APOA5 -12,238T >C, APOA4 Thr347Ser, APOC3 $-482 \mathrm{C}>\mathrm{T}$ and APOC $3238 C>\mathrm{G}($ Sstl) polymorphisms were analyzed in a representative population sample of 3138 men and women from France, including 932 individuals with metabolic syndrome and 2206 without metabolic syndrome, as defined by the NCEP criteria.

Results: Compared with homozygotes for the common allele, the odds ratio (OR) $[95 \% \mathrm{Cl}]$ for metabolic syndrome was I.30 [I.03-I.66] $(p=0.03)$ for APOA5 Trp I 9 carriers, 0.8I [0.69-0.95] ( $p$ $=0.01)$ for APOA5 $-12,238 \mathrm{C}$ carriers and $0.84[0.70-0.99](p=0.04)$ for APOA4 Ser347 carriers. Adjustment for plasma triglycerides, (but not for waist girth, HDL, blood pressure or glycemia the other components of metabolic syndrome) abolished these associations and suggests that triglyceride levels explain the association with metabolic syndrome. There was no association between the APOC3 $-482 C>T$ or APOC $3238 C>G$ polymorphisms and metabolic syndrome. The decreased risk of metabolic syndrome observed in APOA5 - I2,238C and APOA4 Ser347 carriers merely reflected the fact that the $A P O A 5 \operatorname{Trp} 19$ allele was in negative linkage disequilibrium with the common alleles of APOA5 -12,238T >C and APOA4 Thr347Ser polymorphisms.
\end{abstract}

Conclusion: The APOA5 Trp 19 allele increased susceptibility to metabolic syndrome via its impact on plasma triglyceride levels. 


\section{Background}

Metabolic syndrome is a complex disease characterized by the ustering of several metabolic disorders $[1,2]$. Excess body weight, insulin resistance, altered plasma lipid levels $\&$ glucose homeostasis and increased blood pressure are the principal components of this cluster. Environmental influences (such as low physical activity and an inappropriate diet) play a major role in the development of metabolic syndrome. Furthermore, familial aggregation of metabolic disorders has been reported and suggests a genetic contribution to the etiology of this syndrome [35]. Accordingly, it has been reported that genetic variability at several loci is associated with an increased risk of metabolic syndrome [6-12].

The APOA5 gene is located on chromosome 11q23 and codes for a 369 amino-acid protein secreted by the liver [13]. In the plasma, APOA5 is bound to triglyceride-rich and high-density lipoproteins. APOA5 reduces plasma triglyceride levels by inhibiting VLDL-triglyceride production and stimulating LPL-mediated triglyceride hydrolysis [14-18]. In vitro, insulin decreases APOA5 promoter activity - suggesting that the hormone may affect triglyceride levels via an interaction with APOA5 [19]. Given its role in triglyceride metabolism and its interaction with insulin, the APOA5 gene is a candidate gene for metabolic syndrome.

Several common single nucleotide polymorphisms (SNPs) have been described in the APOA5 gene locus; these include a $\mathrm{T}>\mathrm{C}$ substitution at $-12,238$ (SNP4), a T>C substitution at -1131 (SNP3; rs662799) and a C>G mutation at the first base of codon 19 (rs3135506; c.56C>G) that changes a serine (Ser) residue into a tryptophan (Trp) [20]. An association between the APOA5 Ser19Trp polymorphism and plasma triglycerides has been reported in several population samples $[13,20-23]$. In contrast, an association between the APOA5 -12,238T>C polymorphism and plasma lipid variables has not been consistently observed. Together with APOA4, APOC3 and APOA1, the APOA5 gene lies within an expanded gene cluster $[13,24]$. Strong linkage disequilibrium (LD) has been detected for SNPs in this cluster [24]. The APOA5 Ser19Trp and APOC3 3238C >G SNPs (Sst1; rs5128; c.386C>G) both tag common haplotypes in this cluster haplotypes that may have independent effects on plasma triglycerides.

The goal of the present study was to assess the relative contribution of SNPs in the APOA5/A4/C3/A1 locus to the risk of metabolic syndrome by analyzing a limited number of representative SNPs within the cluster and a large population-based sample from 3 areas of France.

\section{Methods Subjects}

Participants were recruited as part of the WHO-MONICA population survey conducted from 1995 to 1997 in three different parts of France: the Lille Urban Community in northern France $(n=1168)$, the Bas-Rhin county in eastern France $(n=1117)$ and the Haute-Garonne county in southern France $(n=1176)$. The protocol was approved by the appropriate independent ethics committee in each center. Subjects (aged 35-64) were randomly selected from electoral rolls after stratification by town size, gender and age in order to obtain 200 participants for each gender and each 10-year age group (WHO-MONICA Project protocol) [25]. A total of 1746 men and 1715 women completed the recruitment procedure. Subsequently, 323 subjects were excluded due to missing data on at least one criterion for metabolic syndrome.

After providing written informed consent, participants filled out a standard questionnaire and physical measurements were taken by a specially trained nurse. The questionnaire covered questions on socioeconomic factors, physical activity, alcohol consumption, smoking status, personal \& family medical history and current medications taken (if any). The level of leisure-time physical activity during was categorized as follows: none, light (light physical activity almost every week), intense (at least 20 minutes of intense physical activity more than once a week). In terms of smoking exposure, subjects were categorized as never-smokers, former smokers and current smokers (i.e. subjects reporting at least one cigarette per day). Total alcohol intake was expressed as the sum of $\mathrm{ml}$ alcohol per week from wine, beer, cider and spirits. Alcohol consumption was categorized in 4 Q1-Q4 classes for men [ $\leq 100 \mathrm{ml} /$ week; $100-250 \mathrm{ml} /$ week; $250-500 \mathrm{ml} /$ week; $>500 \mathrm{ml} /$ week] and for women $[0 \mathrm{ml} /$ week; 0-60 $\mathrm{ml} /$ week; 60-190 ml/week; > $190 \mathrm{ml} /$ week]. Educational level was assessed by counting the number of years of schooling and classifying the values into 3 categories: primary, secondary/technical and university. Anthropometric measurements included body weight (rounded to the nearest even decimal), waist girth (at a level midway between the lower rib margin and the iliac crest, to the nearest $0.5 \mathrm{~cm}$ ) and were performed on subjects in light clothing without shoes. Body mass index was calculated according to the Quetelet equation.

Diabetic subjects were identified by fasting glycemia $\geq 126$ $\mathrm{mg} / \mathrm{dl}$ or antidiabetic treatment. Abdominal obesity was defined by waist girth $\geq 102 \mathrm{~cm}$ in men and $\geq 88 \mathrm{~cm}$ in women. Metabolic syndrome was defined (according to the NCEP III recommendations [26]) by the presence of at least 3 or more of the following abnormalities: waist girth 
$\geq 102 \mathrm{~cm}$ in men and $\geq 88 \mathrm{~cm}$ in women, triglycerides $\geq 1.7$ $\mathrm{mmol} / \mathrm{L}$ or treatment with fibrates, HDL-cholesterol $<1.04 \mathrm{mmol} / \mathrm{L}$ in men and $<1.29 \mathrm{~mol} / \mathrm{L}$ in women, blood pressure $\geq 130 / 85 \mathrm{~mm} \mathrm{Hg}$ or treatment with blood pressure-lowering medications and fasting glucose $\geq 6.1$ mmol/L or type 2 diabetes. Additional analyses were performed using the International Diabetes Federation (IDF) definition [27] of metabolic syndrome. This definition is based on the same criteria as the NCEP definition except that (i) increased waist girth is a mandatory criteria and (ii) the waist cut-offs are 94 and $80 \mathrm{~cm}$ for European men and women, respectively.

\section{Laboratory methods}

A $20 \mathrm{~mL}$ blood sample was drawn into a disodium EDTA tube (after the subjects had fasted for at least 10 hours), stored at room temperature and centrifuged within 4 hours. All measurements were performed in a central laboratory (Purpan Hospital, Toulouse, France). Cholesterol and triglyceride levels were measured using enzyme assays (Boehringer Mannheim, Mannheim, Germany). High density lipoprotein (HDL) cholesterol was measured after sodium phosphotungstate/magnesium chloride precipitation (Boehringer Mannheim, Mannheim, Germany). Glucose was measured using the standard glucose hexokinase method (DuPont Dimension, Brussels, Belgium). The APOA5 Ser19Trp polymorphism was assessed in an Amplifluor $^{\circledast}$ genotyping system using the following probes: APOA5-F1: 5' GAAGGTGACCAAGTTCATGCTTCCCTCTCCACAGCGTTTTC-3'; APOA5-F2: 5'GAAGGTCGGAGTCAACGGATTTCCCTCTCCACAGCGT TTTG-3' and APOA5-R: 5'-TGAAGTAGTCCCAGAAGCCTTT-3'. In the sample, $1.6 \%$ was undetermined. Likewise, the APOC3 3238C>G (SstI) polymorphism was assessed using the following probes: APOC3-F1: 5'GAAGGTCGGAGTCAACGGATTACCTGCCTATCCATC-

CTGCC-3', APOC3-F2: 5'-GAAGGTGACCAAGTTCATGCTACCTGCCTATCCATCCTGCG-3' and APOC3-R: 5'GCACTGAGAATACTGTCCCTTT-3'. In the sample, 3.5\% was undetermined. The APOC3 -482C>T (rs2854117) polymorphism was also assessed in an Amplifluor ${ }^{\circledR}$ system using the following probes: APOC3-F1: 5'GAAGGTGACCAAGTTCATGCTAGTAAAGGCACAGAAGACCG-3', APOC3-F2: 5'-GAAGGTCGGAGTCAACGGATTGAGTAAAGGCACAGAAGACCA-3' and APOC3-R: 5'-TGAAACCCAGAGATGGAGGT-3'. In the sample, 3.8\% was undetermined. The APOA4 Thr347Ser polymorphism was assessed as previously described [28]. The APOA5 $12,238 \mathrm{~T}>\mathrm{C}$ polymorphism was genotyped with an RFLPbased method using the following primers: forward 5'GTGCCTGTCACCACCGTTTG-3' and reverse 5'ATGCATTAGCCTCTGCTGTTC-3' (generating a forced restriction site for HaeIII). The 162 bp PCR fragment was cut by HaeIII into 2 fragments (141 and $21 \mathrm{bp}$ ) for the T allele, whereas a single uncut product resulted for the $\mathrm{C}$ allele. Products were resolved on 3\% agarose gels. For each method, around $10 \%$ of the samples were genotyped twice and no discordant genotypes were observed.

\section{Statistical analyses}

General linear models (GLM) and chi-square tests were used to compare the clinical and biological characteristics of subjects with or without metabolic syndrome. Furthermore, GLM and Chi-square tests were used to compare centers in terms of mean values and the distributions of variables and genotypes, respectively. A GLM adjusted for age, gender and center was used to compare genotype groups in terms of metabolic syndrome criteria. Subjects receiving a treatment that may have affected the level of the variable were excluded from these analyses. Chisquare tests and logistic regression analyses were used to test the association between the various genotypes and metabolic syndrome. For logistic regression analyses, carriers of at least one minor allele were compared with subjects who were homozygous for the frequent allele (dominant model), while adjusting for age, gender and center. The threshold for statistical significance was set to $5 \%$. Linkage disequilibrium between loci was tested using a log-likelihood-ratio test [29]. Disequilibrium was expressed in terms of normalized difference $D^{\prime}=D / D_{\max }$ or $\mathrm{D} / \mathrm{D}_{\min }[30]$. Haplotype frequencies were estimated using a stochastic version of the expectation-maximization algorithm, as implemented in Thesias software $[31,32]$. Differences in haplotype frequencies when comparing groups of subjects with or without metabolic syndrome were examined using a log-likelihood ratio statistic test, which was computed from the haplotype frequency log-likelihoods for each of the two groups estimated in Thesias.

\section{Results}

Table 1 shows the biological and clinical characteristics of the subjects with $(n=932)$ and without $(n=2206)$ metabolic syndrome as defined by NCEP III. As expected, body mass index, waist girth, triglyceridemia, cholesterolemia, glycemia, systolic and diastolic blood pressure were higher and HDL-cholesterol levels were lower in subjects with metabolic syndrome than in subjects without metabolic syndrome (all p < 0.0001). Subjects with metabolic syndrome were less physically active and had spent less time in education than those without metabolic syndrome. The proportion of smokers was lower in the metabolic syndrome group.

The APOA5 Ser19Trp,APOA5 -12,238T>C, APOA4 Thr347Ser, APOC3 -482C>T and APOC3 3238C>G genotypes did not deviate from Hardy-Weinberg equilibrium. Table 2 shows the linkage disequilibrium matrix in subjects without metabolic syndrome. Overall, the $\left|D^{\prime}\right|$ varied from 0.29 to 1 . The APOA5 and APOA4 SNPs were in the 
Table I: Anthropometric, clinical and biological characteristics of subjects with or without metabolic syndrome

\begin{tabular}{|c|c|c|c|}
\hline & Without metabolic syndrome & With metabolic syndrome & $p$ \\
\hline $\mathrm{n}$ & 2206 & 932 & \\
\hline age $(y)$ & $49.4 \pm 8.4$ & $54.2 \pm 8.1$ & $<0.0001$ \\
\hline men & $1044(47.3)$ & $553(59.3)$ & $<0.0001$ \\
\hline $\mathrm{BMI}\left(\mathrm{kg} / \mathrm{m}^{2}\right)$ & $24.8 \pm 3.6$ & $29.9 \pm 4.7$ & $<0.0001$ \\
\hline Waist $(\mathrm{cm})$ & $85.3 \pm 11.3$ & $101.5 \pm 11.6$ & $<0.0001$ \\
\hline Cholesterol (mmol/l) & $5.8 \pm 1.0$ & $6.1 \pm 1.1$ & $<0.0001$ \\
\hline Triglycerides (mmol/l) & $1.00 \pm 0.52$ & $2.20 \pm 2.32$ & $<0.0001$ \\
\hline $\mathrm{HDL}(\mathrm{mmol} / \mathrm{l})$ & $1.58 \pm 0.43$ & $1.19 \pm 0.34$ & $<0.0001$ \\
\hline Glucose (mmol/l) & $5.20 \pm 0.76$ & $5.95 \pm 1.30$ & $<0.0001$ \\
\hline Diabetes (\%) & 2.4 & 19.5 & $<0.0001$ \\
\hline $\mathrm{SBP}(\mathrm{mm} \mathrm{Hg})$ & $125.7 \pm 15.8$ & $140.6 \pm 16.9$ & $<0.0001$ \\
\hline $\mathrm{DBP}(\mathrm{mm} \mathrm{Hg})$ & $79.0 \pm 10.1$ & $87.9 \pm 11.2$ & $<0.0001$ \\
\hline Smoking & & & 0.032 \\
\hline never & $1069(48.5)$ & $432(46.4)$ & \\
\hline former & $629(28.5)$ & $308(33.0)$ & \\
\hline current & $508(23.0)$ & $192(20.6)$ & \\
\hline Physical activity & & & $<0.0001$ \\
\hline none & $449(20.4)$ & $244(26.3)$ & \\
\hline light & $1058(48.0)$ & $490(52.7)$ & \\
\hline intense & $697(31.6)$ & $195(21.0)$ & \\
\hline Alcohol intake & & & 0.0013 \\
\hline QI & $760(34.5)$ & $303(32.5)$ & \\
\hline Q2 & $574(26.0)$ & $20 \mathrm{I}(2 \mathrm{I} .6)$ & \\
\hline Q3 & $535(24.3)$ & $24 \mid(25.9)$ & \\
\hline Q4 & 337 (I5.2) & $187(20.0)$ & \\
\hline Educational level & & & $<0.0001$ \\
\hline low & $433(19.6)$ & $312(33.5)$ & \\
\hline intermediate & $952(43.2)$ & $410(44.0)$ & \\
\hline high & $821(37.2)$ & $210(22.5)$ & \\
\hline
\end{tabular}

Values are means $\pm \mathrm{SD}$ or $\mathrm{n}(\%)$.

BMI : body mass index; HDL : high density lipoprotein ; SBP/DBP : systolic and diastolic blood pressure ; alcohol intake is in quartiles.

same haplotype block ( $\left.\left|D^{\prime}\right| \geq 0.85\right)$; however, due to differences in minor allele frequencies, the $\mathrm{r}^{2}$ values were almost nil. Within this block, both APOA5 -12,238T>C and APOA4 Thr347Ser SNPs were in negative disequilibrium with the APOA5 Ser19Trp SNP. Both APOC3 SNPs were within the same linkage disequilibrium block $\left(D^{\prime}=\right.$ 0.91). Lastly, the APOA4 Thr347Ser SNP was in negative linkage disequilibrium with the APOC3 3238C>G SNP
$\left(D^{\prime}=-0.92\right)$ and thus showed some overlap with the APOC3 haplotype block.

Table 3 shows the metabolic syndrome-related anthropometric, biological and clinical parameters in subjects without metabolic syndrome and as a function of the 5 polymorphisms. Multivariate analyses (adjusted for age, gender and center) revealed that mean triglyceride levels were significantly higher in APOA5 Trp19 ( $\mathrm{p}<0.0001$; $\mathrm{p}$

Table 2: $D$ ' and $r^{2}$ values for linkage disequilibrium among polymorphisms in subjects without metabolic syndrome

\begin{tabular}{|c|c|c|c|c|c|}
\hline & APOA5 Ser19Trp & APOA5 - I2,238 T>C & APOA4 Thr347Ser & APOC3 $-482 C>T$ & APOC3 $3238 C>G$ \\
\hline APOA5 Ser19Trp & - & -0.85 & -1 & -0.73 & -0.41 \\
\hline APOA5 - I2,238 T>C & 0.02 & - & 0.95 & 0.29 & -0.58 \\
\hline APOA4 Thr347Ser & 0.02 & 0.40 & - & 0.45 & -0.92 \\
\hline APOC3 -482 C>T & 0.01 & 0.06 & 0.13 & - & 0.91 \\
\hline APOC3 $3238 C>G$ & 0.00 & 0.02 & 0.02 & 0.23 & - \\
\hline
\end{tabular}

D' upper right corner ; $r^{2}$ lower left corner 
Table 3: Mean levels of metabolic syndrome-related anthropometric, biological and clinical parameters in subjects without metabolic syndrome according to the 5 polymorphisms

\begin{tabular}{|c|c|c|c|c|c|c|}
\hline APOA5 Ser19Trp & Serl9Ser & Ser 19Trp & Trp 19Trp & $p$ & $p^{a}$ & $p^{b}$ \\
\hline Waist (cm) & $85 \pm 11$ & $84 \pm 11$ & $81 \pm 8$ & 0.49 & 0.23 & 0.77 \\
\hline TG $(\mathrm{mmol} / \mathrm{l}) d$ & $1.26 \pm 1.1$ & $1.59 \pm 2.4$ & $2.46 \pm 2.3$ & $<0.0001 c$ & $<0.0001 c$ & $0.07^{c}$ \\
\hline $\mathrm{HDL}(\mathrm{mmol} / \mathrm{l})^{d}$ & $1.48 \pm 0.4$ & $1.49 \pm 0.5$ & $1.2 \pm 0.5$ & 0.56 & 0.78 & 0.59 \\
\hline Glucose $(\mathrm{mmol} / \mathrm{l})$ e & $5.4 \pm I$ & $5.4 \pm 0.8$ & $5.5 \pm 0.4$ & 0.72 & 0.43 & 0.83 \\
\hline $\mathrm{SBP}(\mathrm{mm} \mathrm{Hg})^{f}$ & $129 \pm 17$ & $131 \pm 18$ & $135 \pm 13$ & 0.081 & 0.03 & 0.47 \\
\hline $\mathrm{DBP}(\mathrm{mm} \mathrm{Hg})^{f}$ & $81 \pm 11$ & $82 \pm 10$ & $84 \pm 10$ & 0.082 & 0.03 & 0.39 \\
\hline$A P O A 5-12,238 T>C$ & TT & TC & CC & & & \\
\hline Waist $(\mathrm{cm})$ & $85 \pm 11$ & $85 \pm 11$ & $85 \pm 11$ & 0.49 & 0.23 & 0.15 \\
\hline TG $(\mathrm{mmol} / \mathrm{l}){ }^{d}$ & $1.31 \pm 1.0$ & $1.29 \pm 1.4$ & $1.32 \pm 1.9$ & $0.01 c$ & $0.003^{c}$ & $0.08^{c}$ \\
\hline $\mathrm{HDL}(\mathrm{mmol} / \mathrm{l}) d$ & $1.47 \pm 0.4$ & $1.49 \pm 0.4$ & $1.52 \pm 0.5$ & 0.04 & 0.02 & 0.10 \\
\hline Glucose $(\mathrm{mmol} / \mathrm{l}) \mathrm{e}$ & $5.4 \pm 1$ & $5.4 \pm 1.1$ & $5.3 \pm 0.8$ & 0.31 & 0.52 & 0.15 \\
\hline $\mathrm{SBP}(\mathrm{mm} \mathrm{Hg})^{f}$ & $129 \pm 17$ & $129 \pm 18$ & $129 \pm 17$ & 0.24 & 0.11 & 0.30 \\
\hline $\operatorname{DBP}(\mathrm{mm} \mathrm{Hg})^{f}$ & $81 \pm 11$ & $81 \pm 11$ & $81 \pm 10$ & 0.79 & 0.54 & 0.49 \\
\hline APOA4 Thr347Ser & Thr347Thr & Thr347Ser & Ser347Ser & & & \\
\hline Waist $(\mathrm{cm})$ & $85 \pm 11$ & $86 \pm 11$ & $86 \pm 11$ & 0.64 & 0.71 & 0.39 \\
\hline TG $(\mathrm{mmol} / \mathrm{l}) \mathrm{d}$ & $1.31 \pm 1$ & $1.30 \pm 1.8$ & $1.22 \pm 0.8$ & $0.11 \mathrm{c}$ & $0.04^{c}$ & $0.69 c$ \\
\hline $\mathrm{HDL}(\mathrm{mmol} / \mathrm{l}) \mathrm{d}$ & $1.48 \pm 0.4$ & $1.49 \pm 0.4$ & $1.55 \pm 0.5$ & 0.24 & 0.24 & 0.09 \\
\hline Glucose $(\mathrm{mmol} / \mathrm{l}) \mathrm{e}$ & $5.4 \pm 1.1$ & $5.4 \pm 0.9$ & $5.2 \pm 0.7$ & 0.44 & 0.63 & 0.20 \\
\hline $\mathrm{SBP}(\mathrm{mm} \mathrm{Hg})^{f}$ & $130 \pm 18$ & $129 \pm 17$ & $127 \pm 16$ & 0.09 & 0.03 & 0.82 \\
\hline $\mathrm{DBP}(\mathrm{mm} \mathrm{Hg})^{f}$ & $81 \pm 11$ & $81 \pm 11$ & $81 \pm 10$ & 0.92 & 0.83 & 0.84 \\
\hline APOC3 -482 C>T & CC & CT & TT & & & \\
\hline Waist $(\mathrm{cm})$ & $85 \pm 11$ & $85 \pm 11$ & $85 \pm 11$ & 0.75 & 0.78 & 0.49 \\
\hline TG $(\mathrm{mmol} / \mathrm{l}) \mathrm{d}$ & $1.27 \pm 1$ & $1.34 \pm 1$ & $1.33 \pm 1$ & $0.09 c$ & $0.04 c$ & $0.07^{c}$ \\
\hline $\mathrm{HDL}(\mathrm{mmol} / \mathrm{l}) d$ & $1.47 \pm 0.4$ & $1.49 \pm 0.4$ & $1.5 \mathrm{I} \pm 0.4$ & 0.68 & 0.54 & 0.66 \\
\hline Glucose $(\mathrm{mmol} / \mathrm{l})$ e & $5.4 \pm 1$ & $5.4 \pm 1$ & $5.4 \pm 0.8$ & 0.93 & 0.78 & 0.87 \\
\hline $\mathrm{SBP}(\mathrm{mm} \mathrm{Hg})^{f}$ & $129 \pm 17$ & $129 \pm 18$ & $128 \pm 17$ & 0.47 & 0.45 & 0.28 \\
\hline $\mathrm{DBP}(\mathrm{mm} \mathrm{Hg})^{f}$ & $81 \pm 10$ & $81 \pm 11$ & $81 \pm 11$ & 0.93 & 0.72 & 0.85 \\
\hline APOCЗ $3238 C>G$ & CC & CG & GG & & & \\
\hline Waist $(\mathrm{cm})$ & $85 \pm 11$ & $86 \pm 11$ & $84 \pm 15$ & 0.25 & 0.1 & 0.66 \\
\hline $\mathrm{TG}(\mathrm{mmol} / \mathrm{l}) \mathrm{d}$ & $1.27 \pm 1.4$ & $1.44 \pm 1.2$ & $1.56 \pm 1.2$ & $<0.0001 c$ & $<0.0001 c$ & $0.05 c$ \\
\hline $\mathrm{HDL}(\mathrm{mmol} / \mathrm{l})^{d}$ & $1.49 \pm 0.4$ & $1.47 \pm 0.4$ & $1.43 \pm 0.4$ & 0.15 & 0.6 & 0.29 \\
\hline Glucose $(\mathrm{mmol} / \mathrm{l})$ e & $5.4 \pm 1$ & $5.4 \pm 0.8$ & $5.5 \pm 0.6$ & 0.55 & 0.35 & 0.79 \\
\hline $\mathrm{SBP}(\mathrm{mm} \mathrm{Hg})^{f}$ & $129 \pm 17$ & $129 \pm 17$ & $134 \pm 2 \mid$ & 0.19 & 0.52 & 0.28 \\
\hline $\operatorname{DBP}(\mathrm{mm} \mathrm{Hg})^{f}$ & $81 \pm 11$ & $81 \pm 11$ & $84 \pm 12$ & 0.12 & 0.37 & 0.21 \\
\hline
\end{tabular}

$P$ values ajusted on age, gender and center; $\mathrm{P}^{\mathrm{a}}$ and $\mathrm{P}^{\mathrm{b}}$ values for a dominant and recessive models, respectively; canalyses on log-transformed data ; BMI : body mass index; TG : triglycerides; HDL : high density lipoprotein ; SBP/DBP : systolic and diastolic blood pressure ; Exclusion of subjects treated : ${ }^{\mathrm{d}}$ with fibrates, e oral antidiabetic or insulin, ${ }^{\mathrm{f}} \mathrm{BP}$ lowering therapy.

$<0.0001$ in a dominant model $), A P O C 3-482 \mathrm{~T}(\mathrm{p}=0.04$ in a dominant model) and APOC3 3238G ( $\mathrm{p}<0.0001 ; \mathrm{p}$ $<0.0001$ in a dominant model) allele carriers. Conversely, mean triglycerides were lower in APOA5 -12,238C ( $\mathrm{p}<$ $0.01 ; \mathrm{p}=0.003$ in a dominant model), APOA4 Ser347 ( $=0.04$ in a dominant model) allele carriers than in noncarriers. The mean HDL-cholesterol value was significantly higher in APOA5 $-12,238 \mathrm{C}(\mathrm{p}=0.04 ; \mathrm{p}=0.02$ in a dominant model) allele carriers than in non-carriers. Systolic and diastolic blood pressures were higher in APOA5 Trp19 carriers ( $\mathrm{p}=0.03$ in a dominant model) and systolic blood pressure was lower in APOA4 Ser347 carriers ( $\mathrm{p}=0.03$ in a dominant model). There were no other statistically significant associations for any of the SNPs. Further adjustment for physical activity, alcohol consumption, smoking habits and educational level yielded similar results (data not shown). These associations were similarly observed in all three centers (i.e. there was no significant interaction with geographical area).

In order to assess the contribution of the genetic variability to plasma triglyceride levels, haplotype analyses were performed in subjects without metabolic syndrome using the 5 SNPs in the following order: APOA5 $(\operatorname{Ser}(\mathrm{C}) 19 \operatorname{Trp}(\mathrm{G}), \quad-12,238 \mathrm{~T}>\mathrm{C}), A P O A 4 \quad$ (Thr(A)347Ser (T)) and APOC3 $(-482 \mathrm{C}>\mathrm{T}, 3238 \mathrm{C}>\mathrm{G})$. Eight haplotypes 
Table 4: Haplotype frequencies and delta values of mean triglycerides in subjects without metabolic syndrome

\begin{tabular}{cccc}
\hline Haplotype & Frequency & $\Delta$ TG & $p$ \\
\hline CTACC & 0.454 & reference & - \\
CCACC & 0.130 & 0.037 & 0.13 \\
CCTTC & 0.118 & 0.003 & 0.93 \\
CTATG & 0.079 & 0.165 & $<10^{-6}$ \\
CCTCC & 0.075 & -0.004 & 0.92 \\
GTACC & 0.050 & 0.165 & $<10^{-6}$ \\
CTATC & 0.043 & -0.021 & 0.71 \\
CCATC & 0.022 & -0.013 & 0.83 \\
\hline
\end{tabular}

Only haplotypes with a frequency above $1 \%$ are represented. Order of the SNPS: APOA5 (Ser(C)19Trp(G), 12,238T >C), APOA4 ( Thr(A)347Ser(T)) and APOC3 (-482C $>T, 3238 C>G)$. $P$ values adjusted for age, gender and center.

had an estimated frequency of above 1\% (accounting for more than $97 \%$ of the existing haplotypes) and were used in the analyses (Table 4). The overall association between haplotypes and triglyceride levels was highly significant ( $\mathrm{p}$ $\left.<4 \times 10^{-8}\right)$. By reference to the most common haplotype (CTACC; estimated frequency: 0.45), two haplotypes were significantly associated with raised plasma triglycerides. The GTACC haplotype (estimated frequency: 0.05) that carried the APOA5 Trp19 allele on the common background was associated with a $30 \%$ increase in triglyceride levels $\left(\mathrm{p}<10^{-6}\right)$. The other haplotype (CTATG) carried both the APOC3 $-482 \mathrm{~T}$ and $3238 \underline{\mathrm{G}}$ minor alleles on the common genetic background (estimated frequency: 0.08) and was also associated with a $30 \%$ increase in triglyceride levels $\left(\mathrm{p}<10^{-6}\right)$.

Table 5 shows the genotype distribution of the 5 SNPs in subjects with or without metabolic syndrome. The two groups of subjects differed in terms of the genotype distri-

Table 5: Genotype distributions in subjects with or without metabolic syndrome (MS)

\begin{tabular}{lcccc}
\hline SNP & & & & $P$ \\
\hline APOA5 Ser19Trp & Ser19Ser & Ser 19Trp & Trp 19Trp & \\
Without MS & $1960(88.8)$ & $241(10.9)$ & $5(0.2)$ & 0.10 \\
With MS & $807(86.6)$ & $120(12.9)$ & $5(0.5)$ & \\
APOA5 - I2,238 T>C & TT & CT & CC & \\
Without MS & $912(41.3)$ & $995(45.1)$ & $299(13.5)$ & 0.026 \\
With MS & $413(44.3)$ & $424(45.5)$ & $95(10.2)$ & \\
APOA4 Thr347Ser & Thr347Thr & Thr347Ser & Ser347Ser & \\
Without MS & $1412(64.0)$ & $702(31.8)$ & $92(4.2)$ & 0.034 \\
With MS & $627(67.3)$ & $282(30.3)$ & $23(2.5)$ & \\
APOC3 -482 C>T & CC & CT & TT & \\
Without MS & $1148(52.0)$ & $894(40.5)$ & $164(7.4)$ & 0.26 \\
With MS & $508(54.5)$ & $349(37.4)$ & $75(8.0)$ & \\
APOC3 3238 C>G & CC & CG & GG & \\
Without MS & $1799(81.5)$ & $388(17.6)$ & $19(0.86)$ & 0.71 \\
With MS & $750(80.5)$ & $172(18.4)$ & $10(1.1)$ & \\
& & & & \\
\hline
\end{tabular}

Values are number (\%). bution of APOA5 -12,238T $>\mathrm{C}(\mathrm{p}=0.026)$ and APOA4 Thr347Ser $(\mathrm{p}=0.034)$ SNPs. The adjusted odds ratio (OR) and 95\% confidence interval (CI) for metabolic syndrome in carriers of at least one minor allele (compared with subjects homozygous for the frequent allele) are presented in Table 6. The APOA5 Trp19 allele conferred an increased risk of metabolic syndrome $(\mathrm{p}=0.03)$. In contrast, the APOA5 $-12,238 \mathrm{C}(\mathrm{p}=0.01)$ and the APOA4 Ser347 $(\mathrm{p}=0.04)$ alleles were associated with a lower risk of metabolic syndrome. Adjustment for plasma triglycerides but not for other metabolic syndrome criteria (i.e. waist girth, HDL, glycemia and systolic blood pressure) abolished these associations. Lastly, there was no significant association between the APOC3 $-482 \mathrm{C}>\mathrm{T}$ and $3238 \mathrm{C}>\mathrm{G}$ polymorphisms and metabolic syndrome. Using the IDF criteria for metabolic syndrome, the ORs [95\%CI] for metabolic syndrome were similar in magnitude (data not shown).

Haplotype analyses using the 5 SNPs were performed to assess the relationship with metabolic syndrome. The overall association between the haplotypes and metabolic syndrome was statistically significant $(\mathrm{p}=0.011)$. With respect to the most common haplotype (CTACC), 2 haplotypes were significantly associated with metabolic syndrome. The GTACC haplotype (carrying the APOA5 Trp19 allele on the common background) was associated with a $33 \%$ greater risk of metabolic syndrome $(\mathrm{p}<0.005)$. The other haplotype (CCTTC, carrying the APOA5 12,238C, the APOA4 Ser347 and the APOC3 -482T alleles) was associated with a $26 \%$ reduction in metabolic syndrome risk $(\mathrm{p}=0.03)$. Since the APOC 3 and APOA5 SNPs are in different haplotype blocks, the association with metabolic syndrome was tested for the two blocks independently. Using only the SNPs of APO5 (Ser19Trp, -12,238T>C) and APOA4 Thr347Ser, we found 4 possible haplotypes with a frequency of above $1 \%$. When compared with the common haplotype (CTA; estimated frequency: 0.58), the GTA (estimated frequency 0.05) and the CCT (estimated frequency 0.19 ) haplotypes were respectively associated with a $33 \%$ greater risk $(\mathrm{p}<0.02)$ and a $17 \%$ lower risk (p $=0.034$ ) of metabolic syndrome. Both associations disappeared after adjustment for triglyceride levels. In contrast,

Table 6: Odds ratios for metabolic syndrome in carriers of the minor allele

\begin{tabular}{lccc}
\hline SNP & OR & {$[95 \% \mathrm{Cl}]$} & $P$ \\
\hline APOA5 Ser 19Trp & 1.30 & {$[1.03-1.66]$} & 0.03 \\
APOA5 - I2,238 T>C & 0.81 & {$[0.69-0.95]$} & 0.01 \\
APOA4 Thr347Ser & 0.84 & {$[0.70-0.99]$} & 0.04 \\
APOC3 -482 C>T & 0.89 & {$[0.76-1.06]$} & 0.17 \\
APOC3 3238 C>G & 1.03 & {$[0.84-1.26]$} & 0.76
\end{tabular}

The reference groups were homozygous subjects for the frequent allele. Odds ratios and $p$ values adjusted for age, gender and center. 
haplotype analyses with the two APOC3 SNPs did not reveal any significant association with metabolic syndrome.

\section{Discussion}

The goal of the present study was to assess the contribution of SNPs in the APOA5/A4/C3/A1 cluster to the risk of metabolic syndrome. Our results showed that the APOA5 Trp19 allele was associated with an increased risk of metabolic syndrome and that this association could possibly be explained by an increase in plasma triglyceride levels. Furthermore, the APOA5 -12,238C and APOA4 Ser347 alleles were associated with a lower risk of metabolic syndrome - possibly due to their negative linkage disequilibrium with the APOA5 Trp19 allele. In contrast, the APOC3 $-482 \mathrm{C}>\mathrm{T}$ and $3238 \mathrm{C}>\mathrm{G}$ SNPs did not appear to be related to metabolic syndrome in this French sample.

All 5 SNPs of the cluster tested in this study were associated with triglyceride levels. Compared with the most common haplotype, haplotypes that carried either the APOA5 Trp19 allele only or both the APOC3 -482T and APOC3 3238G alleles were found to be independently associated with elevated triglycerides. These results are consistent with earlier studies in different population samples that reported similar linkage disequilibrium patterns and associations with triglycerides (driven by the APOA5 Trp19 and APOC3 -482T alleles [20]). In addition to elevated triglyceride levels, the APOA5 Trp19 and APOA4 Thr347 alleles were associated with higher blood pressure. A direct effect of APOA5 or APOA4 on blood pressure regulation is unlikely. In contrast, there is experimental evidence to suggest that chronic hypertriglyceridemia leads to endothelium dysfunction, which is associated with an impaired response to vasodilator stimulation [33] and a subsequent decrease in nitric oxide availability [34] - phenomena which may result in increased blood pressure.

The APOA5 Trp19 allele increased the odds ratio for metabolic syndrome, whereas the APOA5 -12,238C and APOA4 Ser347 alleles decreased it. Other studies have reported associations between APOA5 SNPs and metabolic syndrome [8-12]. Our results extend this observation to a different European population sample. The reduced risk of metabolic syndrome observed in carriers of APOA5 -12,238C and APOA4 Ser347 alleles merely reflected the negative linkage disequilibrium existing between these alleles and the APOA5 Trp19 allele. Several lines of evidence suggest that the association with metabolic syndrome is mediated by triglycerides. Firstly, the APOA5 Trp19 allele is associated strongly and independently with triglyceride levels but far less so with blood pressure. Secondly, adjustment for triglycerides (but not for other metabolic syndrome-related criteria) abolished the associations between APOA5 or APOA4 SNPs and the risk of metabolic syndrome. Overall, this suggests that APOA5 and APOA4 genetic variability affected susceptibility to metabolic syndrome.

In contrast, the APOC3 $-482 \mathrm{C}>\mathrm{T}$ and APOC3 $3238 \mathrm{C}>\mathrm{G}$ SNPs did not significantly affect the risk of metabolic syndrome in this sample of French origin, despite significant association with triglyceride level. These SNPs do not belong to the same haplotype as the APOA5 Ser19Trp SNP and have an independent effect on plasma triglycerides, which may explain the difference vis-à-vis APOA5. Furthermore and in contrast to the APOA5 Ser19Trp SNP, the APOC3 SNPs were not associated with another component of metabolic syndrome (such as blood pressure) which could explain this difference. Lastly, the prevalence of hypertriglyceridemia was higher in carriers of the APOA5 Trp19 allele (35\%) than in carriers of the APOC3 $-482 \mathrm{~T}(23 \%)$ or APOC3 3238G (22\%) alleles and thus increased the probability of fulfilling the definition of metabolic syndrome.

This study has both strengths and limitations. It was performed on a representative sample of the French population and therefore avoided potential selection bias for patients and controls. There were more than 900 subjects with metabolic syndrome, which yields enough statistical power to detect an OR of 1.3 for an allele frequency of $10 \%$ at $\alpha=0.05$ and $\beta=90 \%$. Despite this large number of subjects with metabolic syndrome, the statistical power of the study was still not sufficient to account for possible type 1 error due to multiple testing. Therefore, corrections for multiple testing were not applied in the present analysis. In our sample, subjects without metabolic syndrome were, on average, 5 years younger than those with metabolic syndrome. Since metabolic syndrome prevalence increases with age, this may possibly confound (underestimate) the odds ratio associated with the SNPs. However, all our analyses were adjusted for age, in order to account for a possible impact of the latter parameter on the association. Since only a few SNPs per gene were studied, we cannot completely rule out the possibility that other SNPs in different gene loci or specific haplotypes can also contribute to genetic susceptibility to metabolic syndrome. Indeed, other studies have reported associations between APOA5 SNPs and metabolic syndrome [8-12]. Lastly, we used the NCEP working definition of metabolic syndrome; however, additional analyses using the IDF consensus definition generated very similar conclusions and suggested that choice of the definition of metabolic syndrome does not affect the relationship between metabolic syndrome and the SNPs studied here.

\section{Conclusion}

Over the past decades, genetic variability in the APOA5/ A4/C3/A1 cluster has been associated (to varying extents) with variability in plasma lipid, apolipoprotein and lipo- 
protein levels and with an increased risk of cardiovascular disease. The results of the present study further suggest that the association of SNPs in this cluster with metabolic syndrome may be explained by the propensity of plasma triglycerides to increase in APOA5 Trp19 allele carriers.

\section{Competing interests}

The authors declare that they have no competing interests.

\section{Authors' contributions}

DC, AW, PD, JBR, DA, AB, JF and PA participated in the design and coordination of the study. JD and AM carried out the molecular genetic studies, performed the statistical analyses, wrote the paper. All authors read and approved the final manuscript.

\section{Acknowledgements}

These population surveys were supported by unrestricted grants from the Conseil Régional du Nord-Pas de Calais, ONIVINS, Parke-Davies Laboratory, the Mutuelle Générale de I'Education Nationale (MGEN), Groupe Fournier, the Réseau National de Santé Publique, the Direction Générale de la Santé, the Institut National de la Santé Et de la Recherche Médicale (INSERM), the Institut Pasteur de Lille, the Unite d'Evaluation du Centre Hospitalier et Universitaire de Lille, the Centre d'Examen de Santé de Strasbourg, the CPAM de Sélestat and the Fédération Régionale de Cardiologie d'Alsace. "La Fondation de France" is also acknowledged.

\section{References}

I. Reaven GM: Banting lecture 1988. Role of insulin resistance in human disease. Diabetes 1988, 37:1595-1607.

2. Timar O, Sestier F, Levy E: Metabolic syndrome X: A review. Can J Cardiol 2000, I 6:779-789.

3. Mitchell BD, Kammerer CM, Mahaney MC, Blangero J, Comuzzie AG Atwood LD, Haffner SM, Stern MP, MacCluer JW: Genetic analysis of the IRS. Pleiotropic effects of genes influencing insulin levels on lipoprotein and obesity measures. Arterioscler Thromb Vasc Biol 1996, 16:281-288.

4. Hong Y, Pedersen NL, Brismar K, de Faire U: Genetic and environmental architecture of the features of the insulin-resistance syndrome. Am J Hum Genet 1997, 60:143-I52.

5. Chen W, Srinivasan SR, Elkasabany A, Berenson GS: The association of cardiovascular risk factor clustering related to insulin resistance syndrome (Syndrome $X$ ) between young parents and their offspring: the Bogalusa Heart Study. Atherosclerosis 1999, 145:197-205.

6. Dallongeville J, Helbecque N, Cottel D, Amouyel P, Meirhaeghe A: The Gly 16-->ArgI6 and GIn27-->Glu27 polymorphisms of beta2-adrenergic receptor are associated with metabolic syndrome in men. I Clin Endocrinol Metab 2003, 88:4862-4866.

7. Meirhaeghe A, Cottel D, Amouyel P, Dallongeville J: Association between peroxisome proliferator-activated receptor gamma haplotypes and the metabolic syndrome in French men and women. Diabetes 2005, 54:3043-3048.

8. Yamada $Y$, Kato K, Hibino T, Yokoi K, Matsuo H, Segawa T, Watanabe S, Ichihara S, Yoshida H, Satoh K, Nozawa Y: Prediction of genetic risk for metabolic syndrome. Atherosclerosis 2007, |91:298-304.

9. Yamada Y, Ichihara S, Kato K, Yoshida T, Yokoi K, Matsuo H, Watanabe S, Metoki N, Yoshida H, Satoh K, Aoyagi Y, Yasunaga A, Park H, Tanaka $M$, Lee W, Nozawa $Y$ : Genetic risk for metabolic syndrome: examination of candidate gene polymorphisms related to lipid metabolism in Japanese people. I Med Genet 2008, 45:22-28.

10. Grallert H, Sedlmeier EM, Huth C, Kolz M, Heid IM, Meisinger C, Herder C, Strassburger K, Gehringer A, Haak M, Giani G, Kronenberg F, Wichmann HE, Adamski J, Paulweber B, Illig T, Rathmann W:
APOA5 variants and metabolic syndrome in Caucasians. Lipid Res 2007, 48:26|4-262I

II. Kisfali P, Mohas M, Maasz A, Hadarits F, Marko L, Horvatovich K, Oroszlan T, Bagosi Z, Bujtor Z, Gasztonyi B, Wittmann I, Melegh B: Apolipoprotein A5 IVS3+476A allelic variant associates with increased trigliceride levels and confers risk for development of metabolic syndrome in Hungarians. Circ J 2008 , 72:40-43

12. Maasz A, Kisfali P, Horvatovich K, Mohas M, Marko L, Csongei V Farago B, Jaromi L, Magyari L, Safrany E, Sipeky C, Wittmann I, Melegh B: Apolipoprotein A5 T-I I 3 I C variant confers risk for metabolic syndrome. Pathol Oncol Res 2007, 13:243-247.

13. Pennacchio LA, Olivier M, Hubacek JA, Cohen JC, Cox DR, Fruchart JC, Krauss RM, Rubin EM: An apolipoprotein influencing triglycerides in humans and mice revealed by comparative sequencing. Science 200I, 294:169-173.

14. Baroukh N, Bauge E, Akiyama J, Chang J, Afzal V, Fruchart JC, Rubin EM, Fruchart-Najib ], Pennacchio LA: Analysis of apolipoprotein A5, c3, and plasma triglyceride concentrations in genetically engineered mice. Arterioscler Thromb Vasc Biol 2004, 24:I 297-I 302.

I5. Fruchart-Najib J, Bauge E, Niculescu LS, Pham T, Thomas B, Rommens C, Majd Z, Brewer B, Pennacchio LA, Fruchart JC: Mechanism of triglyceride lowering in mice expressing human apolipoprotein A5. Biochem Biophys Res Commun 2004, 3 I 9:397-404

16. Schaap FG, Rensen PC, Voshol PJ, Vrins C, Vliet HN van der, Chamuleau RA, Havekes LM, Groen AK, van Dijk KW: ApoAV reduces plasma triglycerides by inhibiting very low density lipoprotein-triglyceride (VLDL-TG) production and stimulating lipoprotein lipase-mediated VLDL-TG hydrolysis. J Biol Chem 2004, 279:2794 I-27947.

17. van Dijk KW, Rensen PC, Voshol PJ, Havekes LM: The role and mode of action of apolipoproteins CIII and AV: synergistic actors in triglyceride metabolism? Curr Opin Lipidol 2004, I 5:239-246.

18. Merkel M, Loeffler B, Kluger M, Fabig N, Geppert G, Pennacchio LA, Laatsch A, Heeren J: Apolipoprotein AV accelerates plasma hydrolysis of triglyceride-rich lipoproteins by interaction with proteoglycan-bound lipoprotein lipase. J Biol Chem 2005, 280:21553-21560.

19. Nowak M, Helleboid-Chapman A, Jakel H, Martin G, Duran-Sandoval D, Staels B, Rubin EM, Pennacchio LA, Taskinen MR, Fruchart-Najib J, Fruchart JC: Insulin-mediated down-regulation of apolipoprotein A5 gene expression through the phosphatidylinositol 3 kinase pathway: role of upstream stimulatory factor. Mol Cell Biol 2005, 25: 1537-1548.

20. Pennacchio LA, Olivier M, Hubacek JA, Krauss RM, Rubin EM, Cohen JC: Two independent apolipoprotein A5 haplotypes influence human plasma triglyceride levels. Hum Mol Genet 2002, I | :303 |-3038.

21. Hubacek JA: Apolipoprotein A5 and triglyceridemia. Focus on the effects of the common variants. Clin Chem Lab Med 2005, 43:897-902.

22. Chandak GR, Ward KJ, Yajnik CS, Pandit AN, Bavdekar A, Joglekar $\mathrm{CV}$, Fall CH, Mohankrishna P, Wilkin TJ, Metcalf BS, Weedon MN Frayling TM, Hattersley AT: Triglyceride associated polymorphisms of the APOA5 gene have very different allele frequencies in Pune, India compared to Europeans. BMC Med Genet 2006, 7:76.

23. Lai CQ, Parnell LD, Ordovas JM: The APOAI/C3/A4/A5 gene cluster, lipid metabolism and cardiovascular disease risk. Curr Opin Lipidol 2005, 16:153-166.

24. Talmud PJ, Hawe E, Martin S, Olivier M, Miller GJ, Rubin EM, Pennacchio LA, Humphries SE: Relative contribution of variation within the APOC3/A4/A5 gene cluster in determining plasma triglycerides. Hum Mol Genet 2002, I I :3039-3046.

25. The World Health Organization MONICA Project (monitoring trends and determinants in cardiovascular disease): a major international collaboration. WHO MONICA Project Principal Investigators. J Clin Epidemiol I988, 4I : I05-I I 4.

26. Grundy SM, Cleeman JI, Daniels SR, Donato KA, Eckel RH, Franklin BA, Gordon DJ, Krauss RM, Savage PJ, Smith SC Jr, Spertus JA, Costa $F$ : Diagnosis and management of the metabolic syndrome. An American Heart Association/National Heart, Lung, and Blood Institute Scientific Statement. Executive summary. Cardiol Rev 2005, 13:322-327. 
27. Alberti KG, Zimmet $P$, Shaw J: The metabolic syndrome - a new worldwide definition. Lancet 2005, 366: $1059-1062$.

28. Miltiadous G, Hatzivassiliou M, Bashiardes E, Bairaktari E, Cariolou MA, Elisaf M: Genetic polymorphisms of the apolipoprotein AIV in a Greek population and their relation to plasma lipid and lipoprotein levels. Clin Genet 2002, 62:208-2I3.

29. Slatkin M, Excoffier $L$ : Testing for linkage disequilibrium in genotypic data using the Expectation-Maximization algorithm. Heredity 1996, 76:377-383.

30. Lewontin RC: The interaction of selection and linkage. I. General considerations; heterotic models. Genetics 1964, 49:49-67.

31. Tregouet DA, Barbaux S, Escolano S, Tahri N, Golmard JL, Tiret L, Cambien F: Specific haplotypes of the P-selectin gene are associated with myocardial infarction. Hum Mol Genet 2002, I I:2015-2023.

32. Tregouet DA, Escolano S, Tiret L, Mallet A, Golmard JL: A new algorithm for haplotype-based association analysis: the Stochastic-EM algorithm. Ann Hum Genet 2004, 68: 165-I77.

33. de Koning L, Merchant AT, Pogue J, Anand SS: Waist circumference and waist-to-hip ratio as predictors of cardiovascular events: meta-regression analysis of prospective studies. Eur Heart J 2007, 28:850-856.

34. Kusterer K, Pohl T, Fortmeyer HP, Marz W, Scharnagl H, Oldenburg A, Angermuller S, Fleming I, Usadel KH, Busse R: Chronic selective hypertriglyceridemia impairs endothelium-dependent vasodilatation in rats. Cardiovasc Res 1999, 42:783-793.

\section{Pre-publication history}

The pre-publication history for this paper can be accessed here:

http://www.biomedcentral.com/1471-2350/9/84/prepub

Publish with Biomed Central and every scientist can read your work free of charge

"BioMed Central will be the most significant development for disseminating the results of biomedical research in our lifetime."

Sir Paul Nurse, Cancer Research UK

Your research papers will be:

- available free of charge to the entire biomedical community

- peer reviewed and published immediately upon acceptance

- cited in PubMed and archived on PubMed Central

- yours - you keep the copyright

Submit your manuscript here:

http://www.biomedcentral.com/info/publishing_adv.asp 